The Astrophysical Journal, 601:214-220, 2004 January 20

(C) 2004. The American Astronomical Society. All rights reserved. Printed in U.S.A.

\title{
THE ELLIPTICITY OF THE DISKS OF SPIRAL GALAXIES
}

\author{
BARbara S. RYDEN \\ Department of Astronomy, Ohio State University, 140 West 18th Avenue, Columbus, OH 43210; ryden@astronomy.ohio-state.edu \\ Received 2003 June 18; accepted 2003 October 3
}

\begin{abstract}
The disks of spiral galaxies are generally elliptical rather than circular. The distribution of ellipticities can be fitted with a lognormal distribution. For a sample of 12,764 galaxies from the Sloan Digital Sky Survey Data Release 1 (SDSS DR1), the distribution of apparent axis ratios in the $i$ band is best fit by a lognormal distribution of intrinsic ellipticities with $\ln \epsilon=-1.85 \pm 0.89$. For a sample of nearly face-on spiral galaxies analyzed by Andersen \& Bershady using both photometric and spectroscopic data, the best-fitting distribution of ellipticities has $\ln \epsilon=-2.29 \pm 1.04$. Given the small size of the Andersen \& Bershady sample, the two distributions are not necessarily inconsistent with each other. If the ellipticity of the potential were equal to that of the light distribution of the SDSS DR1 galaxies, it would produce 1.0 mag of scatter in the Tully-Fisher relation, greater than is observed. The Andersen \& Bershady results, however, are consistent with a scatter as small as 0.25 mag in the Tully-Fisher relation.
\end{abstract}

Subject headings: galaxies: fundamental parameters — galaxies: photometry — galaxies: spiral

\section{INTRODUCTION}

The disks of spiral galaxies are not axisymmetric structures. Spiral arms are an obvious deviation from axisymmetry, as are bars within barred spiral galaxies. In addition, the overall shape of the disk may be elliptical rather than circular. The shape of a stellar disk can be roughly approximated as a triaxial spheroid with principal axes of length $a \geq b \geq c$. A typical stellar disk is relatively thin, with $\gamma \equiv c / a \ll 1$, and mildly elliptical, with $\epsilon \equiv 1-b / a \ll 1$. The exact distribution of ellipticities for the disks of spiral galaxies has long been a subject of debate.

Statistical statements about the distribution of $\epsilon$ can be made by looking at the distribution of apparent axis ratio $q$ for a large population of spiral galaxies. Sandage, Freeman, \& Stokes (1970) investigated a sample of 254 spiral galaxies from the Reference Catalogue of Bright Galaxies (de Vaucouleurs \& de Vaucouleurs 1964). They concluded that the observed axis ratios of the spiral galaxies were consistent with their being a population of circular disks with typical thickness $\gamma \approx 0.25$. Binney \& de Vaucouleurs (1981), using data from the Second Reference Catalogue of Bright Galaxies (de Vaucouleurs, de Vaucouleurs, \& Corwin 1976), concluded that late-type spirals were better fitted by mildly elliptical disks $(\epsilon=0.1)$ than by perfectly circular disks. Using a sample of 13,482 spiral galaxies, Lambas, Maddox, \& Loveday (1992) found that the ellipticity was reasonably well fitted by a Gaussian peaking at $\epsilon=0$ :

$$
f(\epsilon) \propto \exp \left(-\frac{\epsilon^{2}}{2 \sigma_{\epsilon}^{2}}\right)
$$

subject to the constraint $0 \leq \epsilon \leq 1$. The best fit was given by $\sigma_{\epsilon}=0.13$, yielding an average ellipticity of $\langle\epsilon\rangle \approx 0.1$. The nonaxisymmetry of disks, which is signaled by a scarcity of apparently circular galaxies, has been confirmed by other photometric studies (Grosbøl 1985; Fasano et al. 1993; Alam \& Ryden 2002).

Analyses that rely on the measured axis ratios of a large sample of galaxies have certain intrinsic shortcomings. First, they provide only a statistical statement about the distribution of disk ellipticities in the sample examined; they do not determine the axis ratio of any individual galaxy. Moreover, since these studies rely purely on photometry, they provide information about the ellipticity of the starlight emitted by the galaxy. The ellipticity measured in this way is affected by bars, eccentric rings and pseudorings, loosely wound spiral arms, and patchy star formation, and does not necessarily reflect the overall ellipticity of the potential within which the stars are orbiting. These intrinsic shortcomings are avoided by methods using both photometric and kinematic information.

Consider a disk of test particles orbiting in a potential that is elliptical in the disk plane. The closed particle orbits, when the potential ellipticity is small, will be elliptical themselves (Binney 1978). If the potential is logarithmic, producing a flat rotation curve, the ellipticity of the orbits will equal the ellipticity of the potential. If the resulting elliptical disk is seen in projection, the isophotal principal axes will be misaligned with the kinematic principal axes. (The kinematic principal axes can be defined as the axis along which the line-of-sight component of the rotation velocity is a maximum and the axis along which is zero.) Because of the misalignment, there is generally a nonzero velocity gradient along the isophotal minor axis, proportional to $\epsilon \sin 2 \phi$, where $\epsilon$ is the ellipticity of the potential and $\phi$ is the azimuthal viewing angle measured relative to the long axis of the potential (Franx \& de Zeeuw 1992). Studying the velocity field of gas at large radii where the dark matter should dominate the potential typically yields $\epsilon \sin 2 \phi \lesssim 0.07$ (Schoenmakers, Franx, \& de Zeeuw 1997).

By combining integral field spectroscopy with imaging data, Andersen et al. (2001) were able to determine the intrinsic ellipticity for a number of disk galaxies at low inclination. Since the misalignment between the isophotal principal axes and the kinematic principal axes decreases as the inclination increases, the technique of Andersen et al. (2001) can only be usefully applied to galaxies with inclination $i<30^{\circ}$. For a sample of 28 spiral galaxies, Andersen \& Bershady (2002) found that the intrinsic ellipticities were well fitted by a lognormal distribution. The mean ellipticity of the galaxies in 
their sample was $\bar{\epsilon}=0.076$. The method of Andersen and collaborators has its own shortcomings. The sample of galaxies is relatively small. Systematic uncertainties in determining the isophotal and kinematic major axes can mask the signal produced by an elliptical disk (Barnes \& Sellwood 2003). In addition, to ensure that their sample contained only galaxies with small inclination, Andersen et al. (2001) and Andersen \& Bershady (2002) included only galaxies that appeared nearly circular, with $q \geq 0.866$. This selection bias discriminates against intrinsically highly elliptical disks, which have a relatively small probability of appearing nearly circular in projection. Thus, the true distribution of ellipticities will have a higher mean ellipticity and a larger standard deviation than the Andersen-Bershady sample.

In this paper, I combine information from the two types of analysis: the photometry-only method, which has the advantage of large sample size, and the Andersen et al. (2001) method, which has the advantage of including kinematic information, which probes the potential more directly. In $\S 2$, I use the measured apparent axis ratios of $\sim 12,800$ galaxies from the Sloan Digital Sky Survey Data Release 1 to make an estimate of their intrinsic disk ellipticities. In $\S 3$, I reanalyze the disk ellipticities found by Andersen \& Bershady (2002), taking into account the selection bias, to find the underlying distribution of disk ellipticities. I show that the two methods give results that are not mutually inconsistent. In $\S 4$, I discuss the implications of the disk ellipticity, in particular, its relation to the scatter in the Tully-Fisher relation.

\section{THE SDSS SAMPLE}

The Sloan Digital Sky Survey (SDSS) is a digital photometric and spectroscopic survey, which will cover, when completed, roughly one-quarter of the celestial sphere. Data Release 1 (DR1), issued to the astronomical community in 2003 April, consists of 2099 square degrees of imaging data in five photometric bands: $u, g, r, i$, and $z$ (Fukugita et al. 1996; Stoughton et al. 2002). The data processing pipelines for DR1 (described in Stoughton et al. 2002 and Abazajian et al. 2003) fit two models to the two-dimensional image of each galaxy. The first model has a de Vaucouleurs profile (de Vaucouleurs 1948),

$$
I(r)=I_{0} \exp \left[-7.67\left(r / r_{e}\right)^{1 / 4}\right]
$$

which is truncated beyond $7 r_{e}$ and goes smoothly to zero at $8 r_{e}$; the model has some softening within $r_{e} / 50$. The second model has an exponential profile,

$$
I(r)=I_{0} \exp \left[-1.68\left(r / r_{e}\right)\right]
$$

which is truncated beyond $3 r_{e}$ and goes smoothly to zero at $4 r_{e}$. Both models are assumed to have concentric elliptical isophotes with constant position angle $\varphi_{m}$ and axis ratio $q_{m}$. Before each model is fitted to the data, the model is convolved with a double Gaussian fitted to the point-spread function (PSF). Assessing each model with a $\chi^{2}$ fit gives $r_{e}, q_{m}$, and $\varphi_{m}$ for the best-fitting model, as well as $P(\mathrm{deV})$ and $P(\exp )$, the likelihood associated with the best de Vaucouleurs model and the best exponential model, respectively. In addition, each candidate galaxy is fit directly with the point-spread function, yielding $P(\mathrm{PSF})$, the likelihood associated with the PSF fit.

To build a sample of disk galaxies from the SDSS DR1, I selected objects flagged as galaxies whose model fits in the $r$ band satisfied the criteria $P(\exp )>2 P(\mathrm{deV})$ and $P(\exp )>2 P(\mathrm{PSF})$. In addition, each galaxy was required to have a spectroscopic redshift $z<0.1$ to reduce the possibility of weak lensing distorting the observed shape and $z>0.002$ to eliminate a few contaminating foreground objects. The spectroscopic sample of the SDSS DR1 contained 30,184 galaxies satisfying these criteria. Note that I have selected galaxies on the basis of their surface brightness profile (exponential rather than de Vaucouleurs) and not on other criteria such as color or isophote shape. It is generally true, however, that bright early-type galaxies (elliptical and S0) are better fitted by de Vaucouleurs profiles than by exponential profiles and that spiral galaxies are better fitted by exponential profiles (Strateva et al. 2001). Moreover, the spectroscopic sample of the DR1 contains galaxies that are too high in surface brightness to be dwarf ellipticals, which can also have exponential surface brightness profiles (Binggeli \& Cameron 1991). Thus, I assume that the galaxies that I have chosen on the basis of their exponential profiles are disk-dominated spiral galaxies.

The model fits to the exponential galaxies provide one measure of the axis ratio $q$. However, a model with constant position angle $\varphi$ and axis ratio $q$ is not a good approximation to a real spiral galaxy. Fortunately, the SDSS DR1 photometric analysis also provides more robust, model-free measures of the axis ratio. One useful shape measure is $q_{\mathrm{am}}$, the axis ratio determined by the use of adaptive moments. In general, the technique of adaptive moments determines the $n$th order moments of an image using an elliptical weight function whose shape matches that of the object (Bernstein \& Jarvis 2002; Hirata \& Seljak 2003). In practice, the SDSS adaptive moments use a Gaussian weight function $w(x, y)$, which is matched to the size and ellipticity of the galaxy image $I(x, y)$. With the weight function $w(x, y)$ known, the moments can be calculated:

$$
\begin{gathered}
\boldsymbol{x}_{0}=\frac{\int \boldsymbol{x} w(x, y) I(x, y) d x d y}{\int w(x, y) I(x, y) d x d y} \\
M_{x x}=\frac{\int\left(x-x_{0}\right)^{2} w(x, y) I(x, y) d x d y}{\int w(x, y) I(x, y) d x d y}
\end{gathered}
$$

and so forth. The SDSS DR1 provides for each image the adaptive second moments

$$
\begin{gathered}
T=M_{x x}+M_{y y}, \\
e_{+}=\left(M_{x x}-M_{y y}\right) / T,
\end{gathered}
$$

and

$$
e_{\times}=2 M_{x y} / T
$$

The adaptive second moments can be converted into an axis ratio using the relation

$$
q_{\mathrm{am}}=\left(\frac{1-e}{1+e}\right)^{1 / 2}
$$

where $e \equiv\left(e_{+}^{2}+e_{\times}^{2}\right)^{1 / 2}$. The adaptive moments axis ratios computed in this way are not corrected for the effects of seeing. However, the SDSS DR1 also provides the adaptive second moments, $T_{\mathrm{psf}}, e_{+, \mathrm{psf}}$, and $e_{\times, \mathrm{psf}}$, for the point-spread function at the location of the image. These moments can be used to correct for the smearing and shearing due to seeing; such corrections are vital in studying the small shape changes 
resulting from weak lensing (Bernstein \& Jarvis 2002; Hirata \& Seljak 2003). However, to eliminate the need for corrections, I only retain galaxies that are well resolved, with $r_{e}>5 \sqrt{T_{\mathrm{psf}}}$. Of the five bands used by SDSS, the $g, r$, and $i$ bands provide useful morphological information; the low detector sensitivity at $u$ and $z$ and the high background at $z$ make them less useful for study. At $g, N_{\text {gal }}=12,826$ galaxies satisfy my criteria, at $r$, $N_{\text {gal }}=12,751$, and at $i, N_{\text {gal }}=12,764$.

Because the weight function $w(x, y)$ is scaled to the size of the galaxy image, the adaptive moments axis ratio $q_{\mathrm{am}}$ can be thought of as an average axis ratio to which the outer regions of the galaxy, beyond the effective radius, do not contribute significantly. The shape in the outer regions can be found from the shape of the $25 \mathrm{mag} \operatorname{arcsec}^{-2}$ isophote. The SDSS DR1 provides, for each galaxy in each band, the values of $a_{25}$ and $b_{25}$, the long and short semimajor axis length for the isophote at the surface brightness $25 \mathrm{mag} \operatorname{arcsec}^{-2}$. The isophotal axis ratio $q_{25} \equiv b_{25} / a_{25}$ then provides a measure of the galaxy shape in its outer regions. The mean and standard deviation of $a_{25} / r_{e}$ for the galaxies examined in this paper are $2.12 \pm 0.56$ in the $g$ band, $2.56 \pm 0.72$ in the $r$ band, and $2.82 \pm 0.84$ in the $i$ band. Having two different measures of the axis ratio, $q_{\text {am }}$ and $q_{25}$, thus allows comparison of the central shape $\left(q_{\mathrm{am}}\right)$ and the outer shape $\left(q_{25}\right)$. Having measures in three different bands, $g, r$, and $i$, gives some information about the wavelength dependence of the disks' apparent ellipticity. The $i$ band, having the longest wavelength of those studied, will be least affected by dust and by patches of star formation.

The distribution of $q_{\mathrm{am}}$ in the $i$ band is shown as the histogram in Figure 1. The relative scarcity of nearly circular galaxies is the characteristic signature of nonaxisymmetry. To fit the observed distribution of apparent axis ratios, I adopted a model in which the disk thickness $\gamma$ has a Gaussian distribution,

$$
f(\gamma) \propto \exp \left[-\frac{\left(\gamma-\mu_{\gamma}\right)^{2}}{2 \sigma_{\gamma}^{2}}\right],
$$

subject to the constraint $0 \leq \gamma \leq 1$, and the disk ellipticity $\epsilon$ has a lognormal distribution,

$$
f(\epsilon) \propto \frac{1}{\epsilon} \exp \left[-\frac{(\ln \epsilon-\mu)^{2}}{2 \sigma^{2}}\right],
$$

subject to the constraint $\ln \epsilon \leq 0$. After assuming values for the four parameters $\mu_{\gamma}, \sigma_{\gamma}, \mu$, and $\sigma$, a $\chi^{2}$ fit to the data in Figure 1 can be made. I randomly selected a thickness $\gamma$ and ellipticity $\epsilon$ from the distributions in equations (10) and (11). I then randomly selected a viewing angle $(\theta, \phi)$ and computed the resulting apparent axis ratio (Binney 1985)

$$
q=\left[\frac{A+C-\sqrt{(A-C)^{2}+B}}{A+C+\sqrt{(A-C)^{2}+B}}\right]^{1 / 2},
$$

where

$$
\begin{aligned}
& A=\left[1-\epsilon(2-\epsilon) \sin ^{2} \varphi\right] \cos ^{2} \theta+\gamma^{2} \sin ^{2} \theta \\
& B=4 \epsilon^{2}(2-\epsilon)^{2} \cos ^{2} \theta \sin ^{2} \varphi \cos ^{2} \varphi \\
& C=1-\epsilon(2-\epsilon) \cos ^{2} \varphi
\end{aligned}
$$

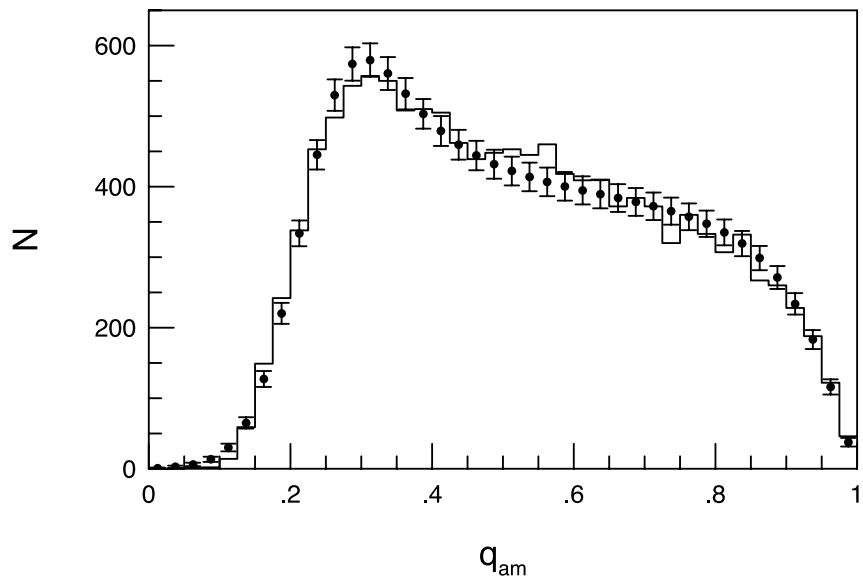

Fig. 1.-Histogram: Distribution of axis ratio $q_{\mathrm{am}}$, using adaptive moments in the $i$ band, for exponential galaxies in the SDSS DR1. Points with error bars: Best-fitting model, assuming a Gaussian distribution of disk thickness and a lognormal distribution of intrinsic disk ellipticity. The best-fitting model has thickness $\gamma=0.222 \pm 0.057$ and ellipticity $\ln \epsilon=-1.85 \pm 0.89$.

By repeating this procedure $N_{\text {gal }}=12,764$ times, I created one realization of the $\left(\mu_{\gamma}, \sigma_{\gamma}, \mu, \sigma\right)$ parameter set. After creating 16,000 realizations, I computed the mean and standard deviation of the expected number of galaxies in each of the 40 bins in Figure 1 and computed a $\chi^{2}$ probability for that particular set of parameters. A search through four-dimensional parameter space revealed that the best fit for $q_{\mathrm{am}}$ in the $i$ band was provided by the set of parameters $\mu_{\gamma}=0.222, \sigma_{\gamma}=$ $0.057, \mu=-1.85$, and $\sigma=0.89$. The resulting $\chi^{2}$ probability was $P=3 \times 10^{-4}$. Formally, this is not a good fit to the data, but more than half the contribution to $\chi^{2}$ comes from the bins on the far left, with $q_{\mathrm{am}}<0.3$. As emphasized by Fasano et al. (1993), the distributions of $\gamma$ and $\epsilon$ for spiral galaxies are almost completely decoupled, in that the distribution of $\gamma$ determines the left-hand side of $f(q)$, while the distribution of $\epsilon$ determines the right-hand side. The model's relatively poor fit at $q_{\mathrm{am}}<0.3$ indicates that the galaxies in the sample have a distribution of thicknesses that is not well fitted by a Gaussian. In any case, the distribution of disk thicknesses in my sample is not the same as the true distribution of disk thicknesses. As shown by Huizinga \& van Albada (1992), a magnitude-limited sample of galaxies such as the SDSS DR1 spectroscopic galaxy sample, which has a limiting (Petrosian) magnitude $r<17.77$, will show a deficit of high-inclination, low- $q$ spiral galaxies. This deficit is due to extinction by dust. In the $B$ band, for instance, an Sc galaxy is 1 to $1.5 \mathrm{mag}$ fainter when seen edge-on than when seen face-on (Huizinga \& van Albada 1992). Although the inclination-dependent dimming is smaller at longer wavelengths and for earlier-type spirals, the fitted disk thickness should be regarded with skepticism. Fortunately for the purposes of this paper, spiral galaxies that appear nearly circular $(q \gtrsim 0.8)$ are nearly face-on, and hence their thickness is almost totally irrelevant; it is the distribution of disk ellipticity $\epsilon$ that determines the shape of $f(q)$ at large $q$.

Table 1 shows the best-fitting model parameters, $\mu_{\gamma}, \sigma_{\gamma}, \mu$, and $\gamma$, for the two different shape measures, $q_{\text {am }}$ and $q_{25}$, and for the three different bands, $g, r$, and $i$. In addition, the best values of $\mu$ and $\gamma$ are plotted as points in Figure 2. Note that going from $g$ band (triangles) to $r$ band (squares) to $i$ band (circles) results in a smaller spread of disk ellipticities (that is, smaller values of $\sigma$ ). Going from $q_{\text {am }}$ (filled symbols) to $q_{25}$ 
TABLE 1

Best-Fitting Models: Gaussian Thickness Distribution, Lognormal Ellipticity Distribution

\begin{tabular}{|c|c|c|c|c|}
\hline Band & $N_{\text {gal }}$ & $\mu_{\gamma} \pm \sigma_{\gamma}$ & $\mu \pm \sigma$ & $P_{\chi^{2}}$ \\
\hline \multicolumn{5}{|c|}{ Adaptive moments $\left(q_{\mathrm{am}}\right)$ Shape Measure } \\
\hline$g \ldots \ldots \ldots \ldots \ldots$ & 12,826 & $0.205 \pm 0.054$ & $-1.79 \pm 1.01$ & $5 \times 10^{-5}$ \\
\hline$r \ldots \ldots \ldots \ldots \ldots$ & 12,751 & $0.216 \pm 0.056$ & $-1.83 \pm 0.93$ & $5 \times 10^{-4}$ \\
\hline$i \ldots \ldots \ldots \ldots \ldots \ldots$ & 12,764 & $0.222 \pm 0.057$ & $-1.85 \pm 0.89$ & $3 \times 10^{-4}$ \\
\hline \multicolumn{5}{|c|}{$25 \mathrm{mag} \operatorname{arcsec}^{-2}$ Isophote $\left(q_{25}\right)$ Shape Measure } \\
\hline$g \ldots \ldots \ldots \ldots \ldots$ & 12,826 & $0.211 \pm 0.056$ & $-2.03 \pm 1.09$ & $2 \times 10^{-2}$ \\
\hline 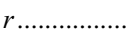 & 12,751 & $0.231 \pm 0.064$ & $-2.07 \pm 0.96$ & $3 \times 10^{-3}$ \\
\hline 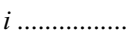 & 12,764 & $0.248 \pm 0.074$ & $-2.06 \pm 0.83$ & $2 \times 10^{-6}$ \\
\hline \multicolumn{5}{|c|}{ Andersen-Bershady } \\
\hline$I \ldots \ldots \ldots \ldots \ldots$ & 28 & $\ldots$ & $-2.29 \pm 1.04$ & 0.996 \\
\hline
\end{tabular}

(open symbols) results in a smaller disk ellipticity (that is, smaller values of $\mu$ ). In the $i$ band, for instance, using $q_{\mathrm{am}}$ for the shape measure results in a best fit lognormal distribution with $\mu=-1.85$ and $\sigma=0.89$; the modal ellipticity for this distribution is 0.071 , the median is $\approx e^{\mu} \approx 0.16$, and the mean is 0.21 . Using $q_{25}$ for the shape measure results in $\mu=-2.06$ and $\sigma=0.83$; the modal ellipticity is 0.064 , the median is $\approx e^{\mu} \approx 0.13$, and the mean is 0.17 . Although the disks are rounder, on average, in the outer regions, they still have a significant ellipticity. Note also the color dependence of the mean thickness $\mu_{\gamma}$; galactic disks are thicker, on average, at longer wavelengths. This dependence reflects the fact that older stellar populations, which have redder colors, have a greater vertical velocity dispersion and hence a greater disk scale height (Wielen 1977; Dehnen \& Binney 1998).

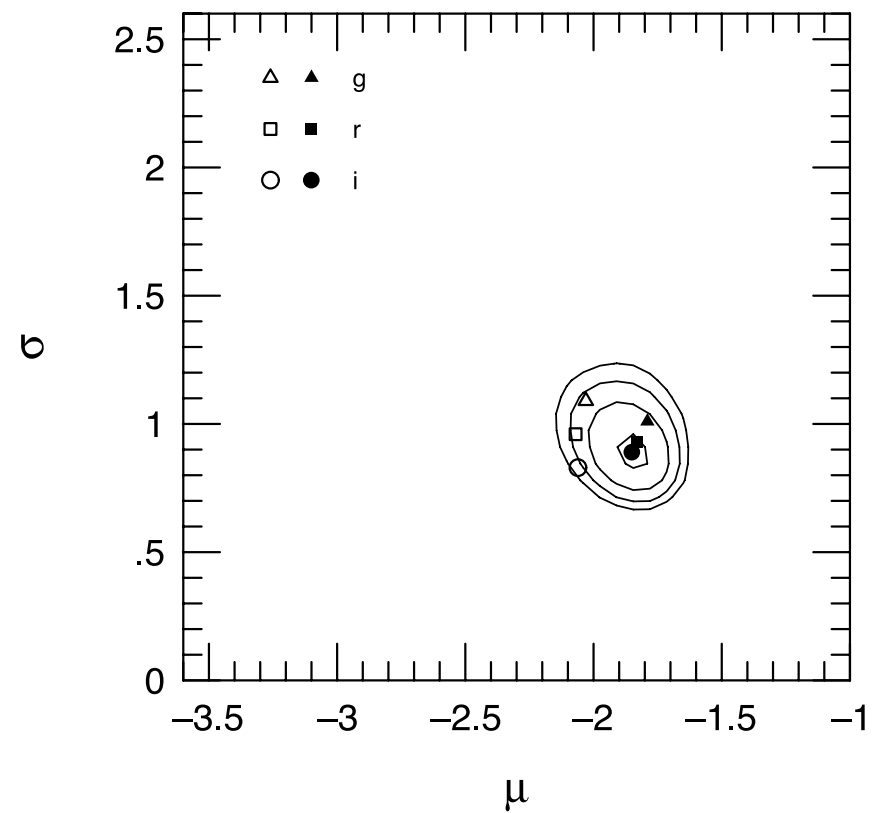

FIG. 2.-Points indicating the best fitting values of $\mu$ and $\sigma$, assuming a lognormal distribution of intrinsic disk ellipticities. Open symbols use $q_{25}$, the axis ratio of the $25 \mathrm{mag} \operatorname{arcsec}^{-2}$ isophote, for the apparent shape measure; filled symbols use $q_{\mathrm{am}}$, the adaptive moments axis ratio, for the apparent shape measure. Solid lines: Isoprobability contours, as measured by a $\chi^{2}$ test applied to the binned data of Fig. 1. Contours are drawn at the levels $\log _{10} P=-4$, $-6,-8$, and -10 , going from the innermost to outermost contour.
The large SDSS DR1 sample of galaxies places strong constraints on the best fitting values of $\mu$ and $\sigma$. To demonstrate how the goodness of fit varies in $(\mu, \sigma)$ parameter space, Figure 2 shows the isoprobability contours for the $q_{\mathrm{am}}$ data in the $i$ band. Note that the contours are drawn at an interval of $\Delta \log _{10} P=2$; the $\chi^{2}$ probability drops rapidly away from the best fit, indicated by the filled circle at the center of the innermost contour. At other wavelengths and using the other shape measure $q_{25}$, the probability falls off with comparable steepness in $(\mu, \sigma)$ parameter space.

The lognormal distribution of equation (11) is not the only functional form to yield an adequate fit to the data. A Gaussian peaking at $\epsilon=0$, as shown in equation (1), provides a comparably good fit to the SDSS DR1 data. In the $i$ band, the bestfitting Gaussian to the $q_{\text {am }}$ data has $\sigma_{\epsilon}=0.26$. The best-fitting Gaussian to the $q_{25}$ data has $\sigma_{\epsilon}=0.21$. The photometry provided by the SDSS DR1 is insufficient to distinguish between a Gaussian distribution of ellipticities, peaking at $\epsilon=0$, and a lognormal distribution, peaking at $\epsilon>0$. Purely photometric studies, it seems, are ill-suited to addressing the question of whether exactly circular disks, with $\epsilon=0$, exist.

\section{THE ANDERSEN-BERSHADY SAMPLE}

Andersen \& Bershady (2002), using the method outlined in Andersen et al. (2001), combined kinematic and $I$-band photometric data to find the disk ellipticity for a sample of 28 nearly face-on disk galaxies; the mean inclination of the galaxies is $i=26^{\circ}$. The distribution of $\ln \epsilon$ determined by Andersen \& Bershady (2002) is shown as the histogram in Figure 3. This distribution is well fitted by a lognormal distribution; the best fit, as found by a $\chi^{2}$ test, has parameters $\mu=-2.80$ and $\sigma=0.81$. However, the best fit to the data in Figure 3 is not the best fit to the underlying distribution of disk ellipticities, thanks to the selection criteria used in building the sample. To ensure that only galaxies with small inclination were included, Andersen \& Bershady (2002) selected galaxies with $q \geq 0.866$, corresponding to an inclination $i \leq 30^{\circ}$ for perfectly circular, infinitesimally thin disks.

To fit the distribution of intrinsic ellipticities in the Andersen-Bershady sample, subject to their selection criterion $q \geq 0.866$, I started by assuming that the disk ellipticity has the lognormal form given in equation (11). I further assumed, for simplicity, that the disks are all infinitesimally thin; since the disks in the Andersen-Bershady sample are close to face-on, 


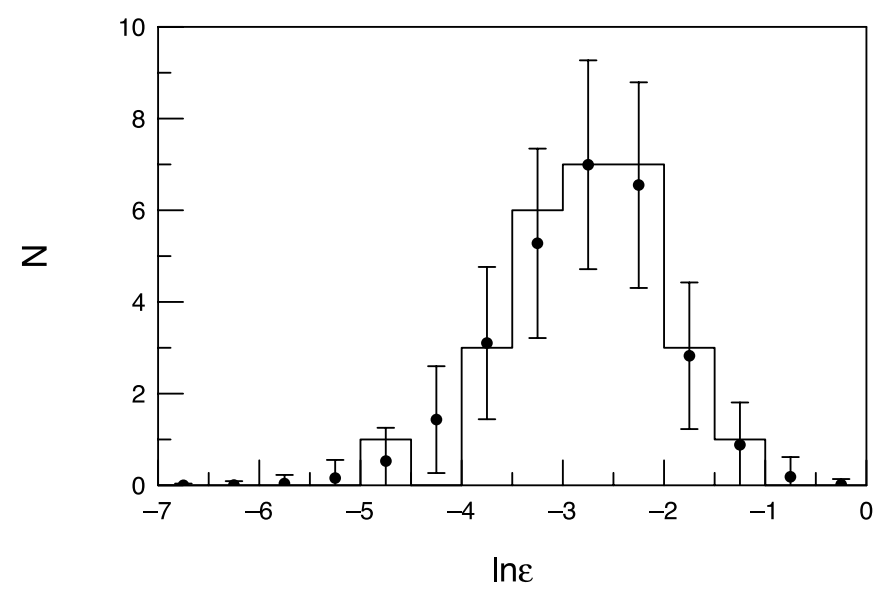

Fig. 3.-Histogram: Distribution of $\ln \epsilon$ for the Andersen-Bershady sample of galaxies. Points with error bars: Best-fitting model, assuming a lognormal distribution of intrinsic disk ellipticity. The best fitting parent distribution has $\ln \epsilon=-2.29 \pm 1.04$. (If the selection criterion $q \geq 0.866$ was ignored, the best fit would have $\ln \epsilon=-2.80 \pm 0.81$.)

their exact thickness does not affect the observed axis ratio. After assuming values for the parameters $\mu$ and $\sigma$, I randomly selected a disk ellipticity $\epsilon$, as well as a viewing angle $(\theta, \phi)$. If the resulting apparent axis ratio, as given by equation (12), was $q \leq 0.866$, I retained it in my sample. If it was flatter than this limit, I discarded it. By repeating this procedure until I had $N_{\text {gal }}=28$ axis ratios, I created one possible realization. After creating 16,000 realizations, I computed the mean and standard deviation in each of the bins in Figure 3. A search through parameter space revealed that the best fit, as measured by a $\chi^{2}$ test, was given by $\mu=-2.29$ and $\sigma=1.04$. The probability of the fit, illustrated by the points and error bars in Figure 3, was $P=0.98$.

The 28 galaxies of Andersen \& Bershady (2002) do not, by themselves, provide a strong constraint on the distribution of intrinsic ellipticities. Figure 4 shows the goodness of fit, as measured by the $\chi^{2}$ probability $P_{\mathrm{AB}}(\mu, \sigma)$. The cross indicates the best fit, and the dotted and solid lines show the isoprobability contours. Note that the $P=0.1$ contour-the innermost dotted line - encloses a large area stretching off the upper right of the plot. That is, a distribution with a large value of $\mu$, corresponding to a very flattened average shape, is acceptable as long as it is paired with a large value of $\sigma$, signifying a wide spread in shapes. Because the Andersen-Bershady sample contains only galaxies that are nearly circular in projection, it is strongly weighted toward galaxies that are nearly circular in their intrinsic shape and thus cannot effectively constrain the high-ellipticity end of $f(\epsilon)$.

A Gaussian peaking at $\epsilon=0$ (see eq. [1]) does not provide a good fit to the Andersen-Bershady sample. The best-fitting Gaussian, with $\sigma_{\epsilon}=0.143$, had a $\chi^{2}$ probability of only $P=0.009$. Thus, although the data of Andersen \& Bershady (2002) does not constrain the high-ellipticity end of $f(\epsilon)$; its discriminatory power at low values of $\epsilon$ weighs strongly against a distribution peaking at $\epsilon=0$.

The kinematic and photometric information exploited by Andersen \& Bershady (2002) is, in some ways, complementary to the purely photometric information included in the SDSS DR1 axis ratios. The nearly face-on galaxies of the AndersenBershady sample constrain the low-ellipticity end of $f(\epsilon)$; the scarcity of nearly circular galaxies in the SDSS DR1 exponential sample (see Fig. 1) constrains the high-ellipticity end of $f(\epsilon)$. The kinematic measurements of Andersen et al. (2001) typically go out to $2-3$ scale lengths $\left(1.2-1.8 r_{e}\right)$. The ellipticities determined by Andersen et al. (2001) and Andersen \& Bershady (2002) can be thought of as average ellipticities over the inner region of the galaxy. Thus, the Andersen-Bershady ellipticities are more directly comparable to the ellipticities found from $q_{\text {am }}$ than those found from the outer axis ratios $q_{25}$. As shown in Figure 4, the best fit using $q_{\text {am }}$ in the $i$ band, indicated by the filled circle, is marginally consistent with the Andersen-Bershady results. Multiplying together the probability fields in Figures 2 and 4 yields a best joint fit of $\mu=$ -1.89 and $\sigma=0.96$. For this set of parameters, the $\chi^{2}$ fit to the Andersen-Bershady data has $P=0.44$, and the fit to the $q_{\mathrm{am}}$ data in the $i$ band has $P=9.5 \times 10^{-5}$.

\section{DISCUSSION}

In this paper, I have considered two quite different ways of determining disk ellipticities. The SDSS DR1 axis ratios are a measure of where the starlight is in a galaxy and hence contain information about bulges, bars, spiral arms, and other nonaxisymmetric structure in the galaxies. By its nature, the analysis of photometric axis ratios finds difficulty in distinguishing between a Gaussian distribution of ellipticities peaking at $\epsilon=0$ and a lognormal distribution peaking at $\epsilon>0$. For determining whether truly circular disks actually exist, examining the apparent axis ratios of disks (even of a large number of disks) is not an effective method to use. By contrast, the approach of Andersen et al. (2001) and Andersen \& Bershady (2002) uses both photometric and kinematic information to find the ellipticity of individual disks. Since Andersen \& Bershady (2002) looked at disks that are nearly circular in projection, their data are ineffective at determining the high-ellipticity end of $f(\epsilon)$.

Although both data sets are reasonably well fit by a lognormal distribution with $\ln \epsilon=-1.89 \pm 0.96$, this distribution should not be engraved on stone as the distribution of disk

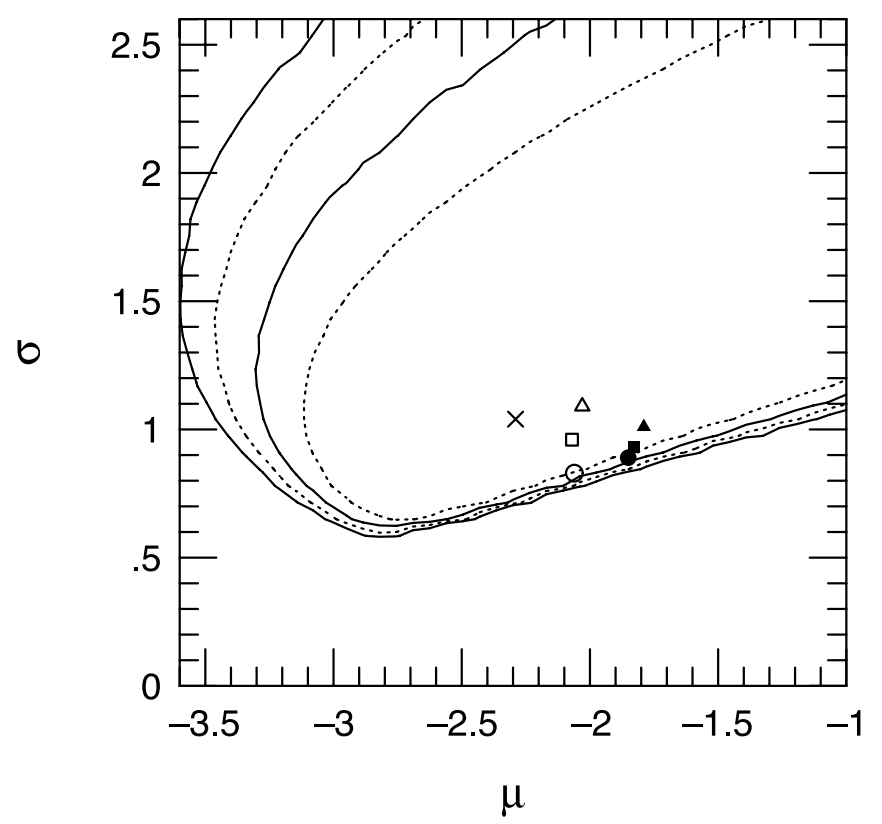

FIG. 4.- Isoprobability contours, as measured by a $\chi^{2}$ test, applied to the binned data of Fig. 3 in $(\mu, \sigma)$ parameter space. Contours are drawn at the levels $\log _{10} P=-1,-2,-3$, and -4 , going from the innermost to outermost contour. The cross indicates the best fit: $\mu=-2.29, \sigma=1.04$. The best fitting points for the SDSS DR1 data are repeated from Fig. 2 for comparison purposes. 
ellipticities in spiral galaxies. Describing a complex structure such as a spiral galaxy with a single number $\epsilon$ (or even two numbers, $\epsilon$ and $\gamma$ ) requires averaging over a great deal of substructure. How one takes the average will affect the value of $\epsilon$ found. For instance, using the adaptive moments shape $q_{\text {am }}$ yields larger values for the ellipticity than using the isophotal shape $q_{25}$. Moreover, observations at different wavelengths result in different values of $\epsilon$.

If a disk of gas and stars is orbiting in a logarithmic potential that is mildly elliptical in the disk plane with $\epsilon_{\phi} \ll 1$, then the integrated line profile from the disk will have a width $W=$ $2 v_{c}\left(1-\epsilon_{\phi} \cos 2 \varphi\right) \sin \theta$ when viewed from a position angle $\varphi$, $\theta$ (Franx \& de Zeeuw 1992). The alteration in the line width, due to noncircular motions in the elliptical potential, will produce a scatter in the observed Tully-Fisher relation between line width and absolute magnitude (Tully \& Fisher 1977). For an ellipticity $\epsilon_{\phi}=0.1$, the expected scatter is $0.3 \mathrm{mag}$ (Franx \& de Zeeuw 1992). (This assumes that the inclination has been determined accurately using kinematic information; if the inclination is determined photometrically, assuming the disk is circular, there will be an additional source of scatter.)

If the potential ellipticity $\epsilon_{\phi}$ is assumed to be drawn from a lognormal distribution, and the embedded disk is viewed from a random angle, the resulting scatter in the Tully-Fisher relation is shown in Figure 5. The best fits for the SDSS DR1 data are superimposed as triangles ( $g$ band), squares ( $r$ band) and circles ( $i$ band). Even in the $i$ band, the best fit to the ellipticity of the disks would produce far more scatter than is seen in the TullyFisher relation. Using $q_{\mathrm{am}}$ as the shape measure (as shown by the filled circle in Fig. 5), 1.0 mag of scatter is predicted. Using $q_{25}$ as the measure (as shown by the open circle in Fig. 5), 0.8 mag of scatter are predicted. The best fit to the AndersenBershady data, shown as the cross in Figure 5, would also produce 0.8 mag of scatter in the Tully-Fisher relation.

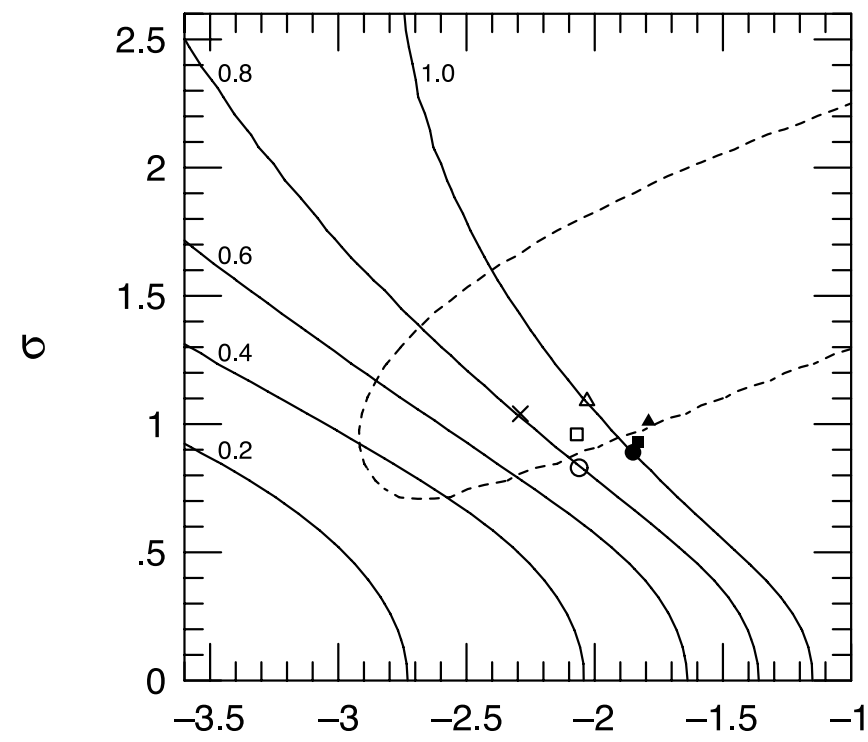

$\mu$

FIG. 5.- Scatter in magnitudes around the mean Tully-Fisher relation for a lognormal distribution of potential ellipticity. The contours are drawn, starting at the lower left, at the levels $0.2,0.4,0.6,0.8$, and 1.0 mag. Dashed line: $50 \%$ probability contour for the Andersen-Bershady data. Cross: Best fit for the Andersen-Bershady data. The open and filled symbols are the best fits for the SDSS DR1 data repeated from Fig. 2.
In contrast, the actual scatter in the Tully-Fisher relation is smaller than 0.8 mag. Courteau (1997) found 0.46 mag of scatter in the optical Tully-Fisher relation when he used as his velocity measure the rotation speed at 2.2 scale lengths $\left(\sim 1.3 r_{e}\right)$, which is about the extent of the kinematic data of Andersen et al. (2001). Verheijen (2001) in his study of spiral galaxies in the Ursa Major cluster found a still smaller scatter. Combining near-infrared $K^{\prime}$ magnitudes with $V_{\text {flat }}$, the rotation speed in the outer, flat part of the rotation curve, Verheijen (2001) found a best fit with zero intrinsic scatter with an upper limit, at the $95 \%$ confidence level, of $0.21 \mathrm{mag}$.

The SDSS DR1 data are clearly inconsistent with such small scatters in the Tully-Fisher relation. The light distribution in the $i$ band cannot reflect the ellipticity of the underlying potential but must be due primarily to nonaxisymmetric structures such as bars, spiral arms, noncircular rings, and so forth. It should be noted that lopsidedness ( $m=1$ distortions) is not uncommon in disk galaxies; Rix \& Zaritsky (1995) found that one third of the galaxies in their sample of nearly face-on spirals had significant lopsidedness at 2.5 scale lengths $\left(\sim 1.5 r_{e}\right)$. Unfortunately, the SDSS DR1 does not provide, among its tabulated parameters, the odd moments that would permit a quantitative estimate of disk lopsidedness.

Although barred galaxies were excluded from the AndersenBershady sample, I made no effort to sift out barred galaxies from my SDSS DR1 sample. The Andersen-Bershady results are not inconsistent with a small scatter in the Tully-Fisher relation. The dashed line in Figure 5 is the $P=0.5$ contour for the Andersen-Bershady sample; that is, every $(\mu, \sigma)$ pair within the dashed line gives a "too good to be true" fit to the sample of Andersen \& Bershady (2002). This contour encloses $(\mu, \sigma)$ pairs that produce as little as $0.32 \mathrm{mag}$ of scatter. At lower probability levels, the $P=0.1$ contour yields as little as $0.28 \mathrm{mag}$ and the $P=0.01$ contour yields as little as $0.25 \mathrm{mag}$ of scatter. In summary, the Andersen-Bershady data are consistent with as little as $0.25 \mathrm{mag}$ of scatter in the Tully-Fisher relation. The Andersen-Bershady data are also consistent with the adaptive moments axis ratios from the SDSS DR1. However, the three sets of information-the AndersenBershady data, the Tully-Fisher scatter (or lack thereof), and the SDSS DR1 axis ratios - are not mutually consistent. The SDSS DR1 axis ratios, if they accurately trace the potential ellipticity, would produce too much scatter in the Tully-Fisher relation.

I thank Matt Bershady, Dave Andersen, Richard Pogge, and Albert Bosma for their helpful and courteous comments. Ani Thakar was an invaluable guide to the sdssQA query tool. The Sloan Digital Sky Survey (SDSS) is a joint project of the University of Chicago, the Institute of Advanced Study, the Japan Participation Group, the Max-Planck-Institute for Astronomy (MPIA), the Max-Planck-Institute for Astrophysics (MPA), New Mexico State University, Princeton Observatory, the United States Naval Observatory, and the University of Washington. Apache Point Observatory, site of the SDSS telescopes, is operated by the Astrophysical Research Consortium (ARC). Funding for the project has been provided by the Alfred P. Sloan Foundation, the SDSS member institutions, the National Aeronautics and Space Administration, the National Science Foundation, the US Department of Energy, the Japanese Monbukagakusho, and the Max Planck Society. The SDSS website is http://www.sdss.org/. 
Abazajian, K., et al. 2003, AJ, 126, 2081

Alam, S. M. K., \& Ryden, B. S. 2002, ApJ, 570, 610

Andersen, D. R., \& Bershady, M. A. 2002, in ASP Conf. Ser. 275, Disks of Galaxies: Kinematics, Dynamics, and Perturbations, ed. E. Athanassoula \& A. Bosma (San Fransisco: ASP), 39

Andersen, D. R., Bershady, M. A., Sparke, L. S., Gallagher, J. S., \& Wilcots, E. M. 2001, ApJ, 551, L131

Barnes, E. I., \& Sellwood, J. A. 2003, AJ, 125, 1164

Bernstein, G. M., \& Jarvis, M. 2002, AJ, 123, 583

Binggeli, B., \& Cameron, L. M. 1991, A\&A, 252, 27

Binney, J. 1978, MNRAS, 183, 779 1985, MNRAS, 212, 767

Binney, J., \& de Vaucouleurs, G. 1981, MNRAS, 194, 679

Courteau, S. 1997, AJ, 114, 2402

Dehnen, W., \& Binney, J. J. 1998, MNRAS, 298, 387

de Vaucouleurs, G. 1948, Ann. d'Astrophys., 11, 247

de Vaucouleurs, G., \& de Vaucouleurs, A. 1964, Reference Catalogue of Bright Galaxies (Austin: Univ. Texas Press)

de Vaucouleurs, G., de Vaucouleurs, A., \& Corwin, H. G. 1976, Second Reference Catalogue of Bright Galaxies (Austin: Univ. Texas Press)
REFERENCES

Fasano, G., Amico, P., Bertola, F., Vio, R., \& Zeilinger, W. W. 1993, MNRAS, 262, 109

Franx, M., \& de Zeeuw, T. 1992, ApJ, 392, L47

Fukugita, M., Ichikawa, T., Gunn, J. E., Doi, M., Shimasaku, K., \& Schneider, D. P. 1996, AJ, 111, 1748

Grosbøl, P. J. 1985, A\&AS, 60, 261

Hirata, C., \& Seljak, U. 2003, MNRAS, 343, 459

Huizinga, J. E., \& van Albada, T. S. 1992, MNRAS, 254, 677

Lambas, D. G., Maddox, S. J., \& Loveday, J. 1992, MNRAS, 258, 404

Rix, H.-W., \& Zaritsky, D. 1995, ApJ, 447, 82

Sandage, A., Freeman, K. C., \& Stokes, N. R. 1970, ApJ, 160, 831

Schoenmakers, R. H. M., Franx, M., \& de Zeeuw, P. T. 1997, MNRAS, 292, 349

Stoughton, C., et al. 2002, AJ, 123, 485

Strateva, I., et al. 2001, AJ, 122, 1861

Tully, R. B., \& Fisher, J. R. 1977, A\&A, 54, 661

Verheijen, M. A. W. 2001, ApJ, 563, 694

Wielen, R. 1977, A\&A, 60, 263 